\title{
Correlation of histological findings with gadolinium enhanced MRI scans during healing of a PHEMA orbital implant in rabbits
}

Lions Eye Institute and Centre for Ophthalmology and Visual Science, University of Western Australia, Australia C R Hicks

$S$ Vijayasekaran

A B Clayton

T V Chirila

G J Crawford

I J Constable

Department of Radiology, Sir Charles Gairdner Hospital, Perth, Australia

I T Morris

M J Fallon

J McAllister

Correspondence to: Dr C R Hicks, Lions Eye Institute, 2 Verdun Street, Nedlands, Western Australia 6009 , Australia.

Accepted for publication 25 November 1998

Celia R Hicks, Ian T Morris, Sarojini Vijayasekaran, Michael J Fallon, Jordana McAllister, Anthony B Clayton, Traian V Chirila, Geoffrey J Crawford, Ian J Constable

\begin{abstract}
Backgroundlaims-To investigate a poly(2-hydroxyethyl methacrylate) (PHEMA) orbital implant with a spongy anterior hemisphere and a smooth gel posterior hemisphere, by histology correlated with magnetic resonance images.

Methods-Following enucleation, eight rabbits received PHEMA implants to which the muscles were directly sutured, and underwent gadolinium enhanced magnetic resonance imaging (MRI) from 3 to 52 weeks. After the rabbits were killed, the implants were removed, cut in a plane corresponding to the scan, and processed for light and electron microscopy.

Results-All eight rabbits retained their implant to the end of the study period without complications. The scans demonstrated muscle attachment to the anterior half of the implant, and enhancement was seen on injection of gadolinium chelate. Histology confirmed muscle attachment, and cellular and vascular ingrowth. Over time, a transformation from reactive inflammatory to relatively non-vascular scar tissue was seen within the implant. Calcium deposits in one implant were detected by imaging and histology.

Conclusion-The implants are readily visualised on MRI. Muscle attachment and fibrovascular ingrowth into the anterior hemisphere are seen, while encapsulation of the posterior hemisphere is minimal. Histological findings confirm the progress of the healing response, with initial inflammation and marked vascularisation, developing later into quiescent scar tissue predominantly of fibroblasts. (Br F Ophthalmol 1999;83:616-621)
\end{abstract}

We have previously reported the development of a novel soft orbital implant made from poly(2hydroxyethyl methacrylate) (PHEMA). ${ }^{12}$ The implant is a sphere of PHEMA comprising a high water content anterior sponge hemisphere and a lower water content posterior gel hemisphere, which are chemically identical and fused at the equator by means of an interpenetration of the polymers, an extremely strong junction. ${ }^{3}$ This implant allows muscles to be sutured directly to its anterior hemisphere, preventing the need for implant wrapping. The anterior hemisphere is biocolonisable, but the posterior resists tissue attachments. During clinical assessment of the implant in 18 animals for up to
32 months to date we have found a low incidence of complications. ${ }^{2}$ No implant has extruded and good motility is seen on clinical examination. We continue to follow these animals to ascertain the long term complication rate.

The present animal study was designed to evaluate the nature and time course of biointegration of the PHEMA implant in order to predict whether long term implant stability can be expected. Histological findings were correlated with magnetic resonance images to demonstrate the extent to which biocolonisation of the implant can be detected by magnetic resonance imaging (MRI) and thus assess the potential usefulness of MRI to detect complications in the postoperative patient. MRI with gadolinium enhancement was selected as the imaging method of choice, having been demonstrated to be superior to technetium-99 bone scans after hydroxyapatite orbital implant placement, ${ }^{4}$ and being able to produce better contrast resolution than computed tomography (CT).

\section{Methods}

PHEMA orbital implants (Fig 1) were manufactured in our laboratories as described previously, ${ }^{5}$ the process involving two step casting followed by cryolathing. Any remnants of toxic monomer were removed by Soxhlet extraction over 24 hours before sterilisation by autoclave and storage in sterile balanced salt solution.

Animal care and procedures conformed with the Australian code of practice for the care and use of animals for scientific purposes. Each of

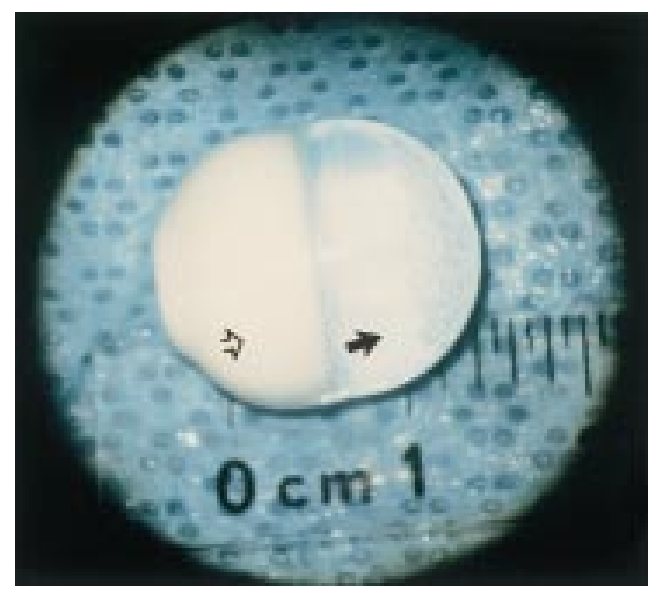

Figure 1 The PHEMA orbital implant. Sponge anterior hemisphere indicated by the open arrow, gel posterior hemisphere by the solid arrow. 


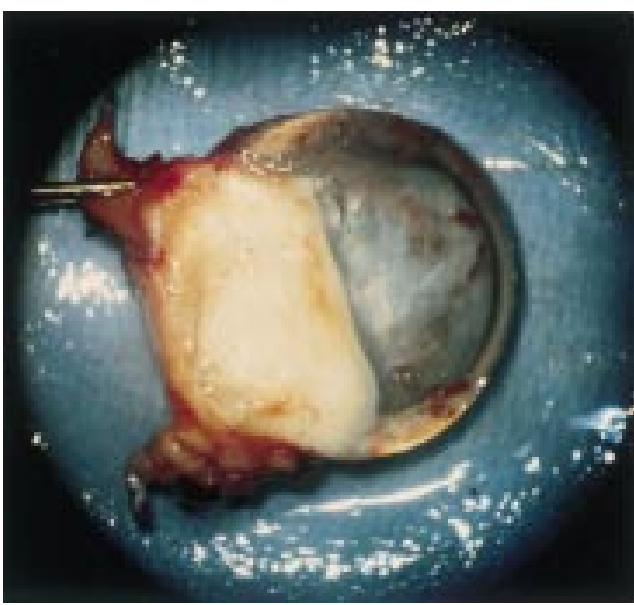

Figure 2 Photograph of hemisected implant (12 months) showing muscle insertions onto the sponge anterior hemisphere of the implant, forceps holding the superior rectus.

eight half lop rabbits underwent right enucleation under halothane/oxygen general anaesthesia. A PHEMA implant was placed within the socket and the vertical extraocular muscles were sutured directly to the anterior sponge hemisphere using $6 / 0$ chromic gut. The lateral recti are small in the rabbit and were not attached. Tenon's layer was closed using a chromic gut purse string and the conjunctiva closed with continuous $6 / 0$ Vicryl. A single subconjunctival injection of gentamicin and dexamethasone was given at the completion of surgery, and intramuscular buprenorphine 0.2 mg was also administered. Chloramphenicol ointment was given once daily for the first postoperative month.

At intervals (one rabbit at 3 weeks, two at 2 months, one at 4 months, two at 6 months, two at 12 months) sedated animals underwent orbital MRI before and after enhancement with gadodiamide (Nycomed, Sydney, Australia) $(0.6 \mathrm{ml} / \mathrm{kg}$ intravenously). MRI was performed using a Siemens Vision 1.5 Tesla scanner (Siemens Medical Imaging, Erlangen, Germany) and an automatically tunable circular receiving coil with a diameter of $35 \mathrm{~mm}$

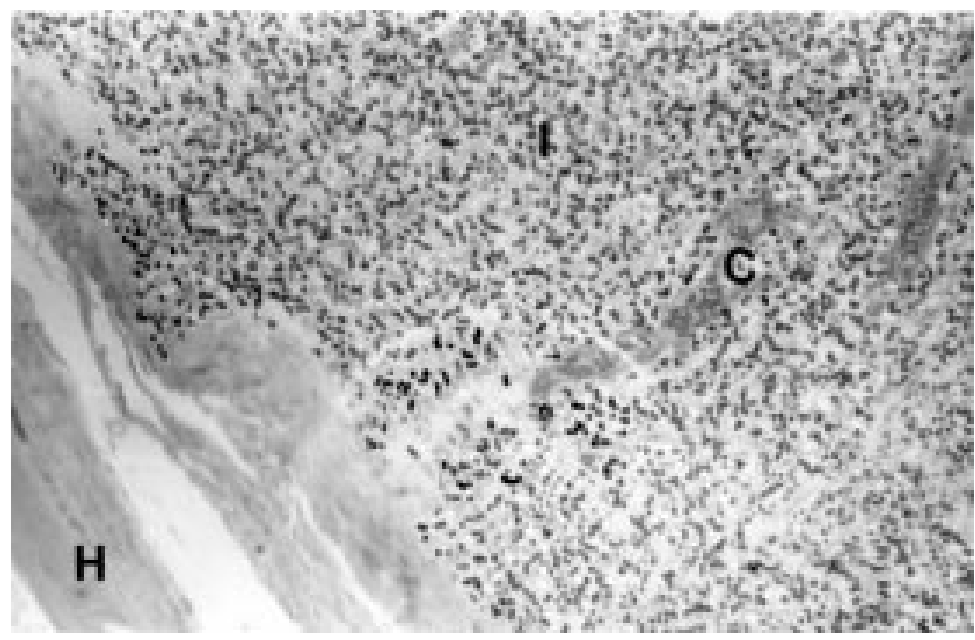

Figure 3 Light micrograph showing attachment of an extraocular muscle to the implant by means of fibrovascular ingrowth from its capsule: implant (I), host (H), and blood capillaries (C), 12 months after implantation. Original magnification $\times 330$.
(Siemens Medical Imaging, Erlangen, Germany). The subjects were scanned in the prone position, with imaging performed in the coronal plane, resulting in "anteroposterior" images through the laterally directed rabbit orbit. Thus, the attachments of the superior and inferior recti to the prosthesis were optimally demonstrated. Imaging factors were as follows: slice thickness $=3 \mathrm{~mm}$; interslice gap $=3.3 \mathrm{~mm}$. T1 weighted $(\mathrm{T} 1 \mathrm{WI})(\mathrm{TR}$ (repetition time $)=400 \mathrm{~ms} ; \mathrm{TE}($ echo time $)=14$ $\mathrm{ms}$ ), T2 weighted fast spin echo (T2WI) (TR $=4000 \mathrm{~ms}$; TE (effective) $=96 \mathrm{~ms}$; ETL (echo train length) $=7$ ) and gadolinium enhanced T1WI and T1WI with fat suppression were obtained. The images were assessed by two of the authors (MJF and JM), who were blind to which treatment the rabbits received, using the following criteria: imaging characteristics of the prosthesis; enhancement at the site of attachment of the superior and inferior recti to the prosthesis, and of the spongy anterior hemisphere (graded using a four point scale of + to ++++ ) and enhancement of the posterior rim of the gel hemisphere of the prosthesis, an indication of capsule formation.

On completion of scanning the animals were killed by intravenous barbiturate injection. The conjunctiva was incised in the fornices and the implant, with adherent tissue, was removed. Vicryl sutures were used to indicate the "top" of the removed implant to allow histological orientation in relation to the scans. The implant was bisected in a plane corresponding with that of the scan, and the cut faces photographed and examined. The strength of attachment of extraocular muscles was assessed by pulling on the cut stumps with forceps.

The implants were fixed in $2.5 \%$ glutaraldehyde in $0.1 \mathrm{M}$ buffer. They were post fixed in osmium tetroxide, dehydrated in graded series of ethanol, and embedded in epoxy resin (Durcupan ACM, Fluka AG, Switzerland) for light microscopy. Semithin $(2 \mu \mathrm{m})$ sections were cut and stained with either toluidine blue or $2 \%$ alizarin red. Ultrathin $(0.1 \mu \mathrm{m})$ sections were also cut and stained with uranyl acetate and lead citrate for transmission electron microscopy (TEM).

\section{Results}

CLINICAL

No implant exposure, extrusion, or migration occurred, and the conjunctiva remained thick and uninflamed. Implant movements, in concordance with the fellow eye, were easily seen. After removal, it was found that the recti were very strongly attached to the implants, and could not be pulled off using forceps (Fig 2). Their connective tissue capsules were seen to be continuous with the fibrovascular ingrowth into the sponge.

\section{HISTOLOGICAL}

Light microscopic examination of the prostheses revealed fibroblasts from the muscle capsule penetrating deeply into the anterior hemispheres (Fig 3). Remnants of suture material persisted within the sponge in several cases. 


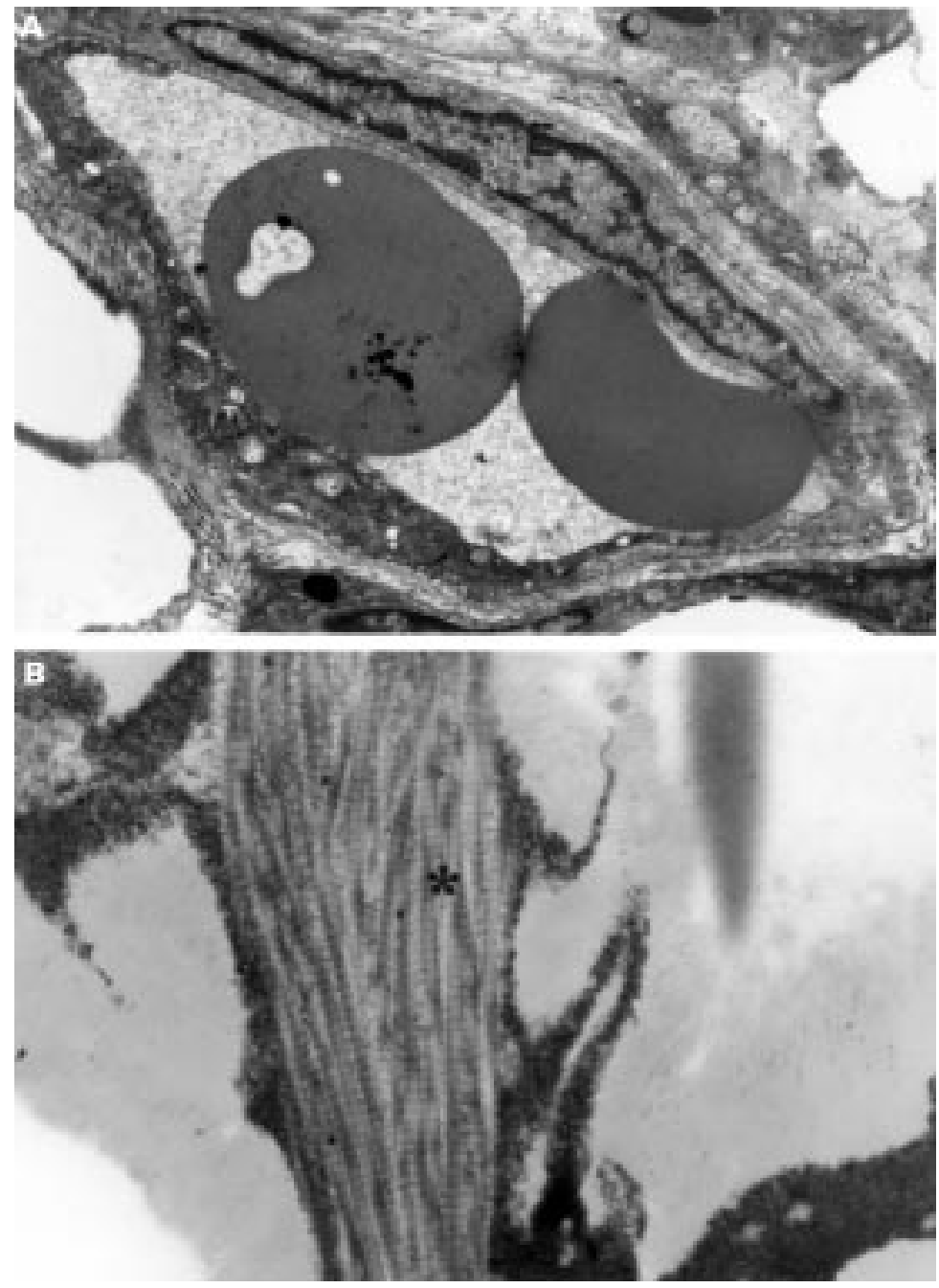

Figure 4 Transmission electron micrographs $(A)$ of a blood capillary, lined with flat, normal looking endothelial cells (E), deep within the sponge of the implant; red blood cells are seen within the lumen of the vessel (original magnification $\times 13000$ ) and $(B)$ of mature collagen fibres (*) that have been laid down in the pores of the implant. Original magnification $\times 15000$.
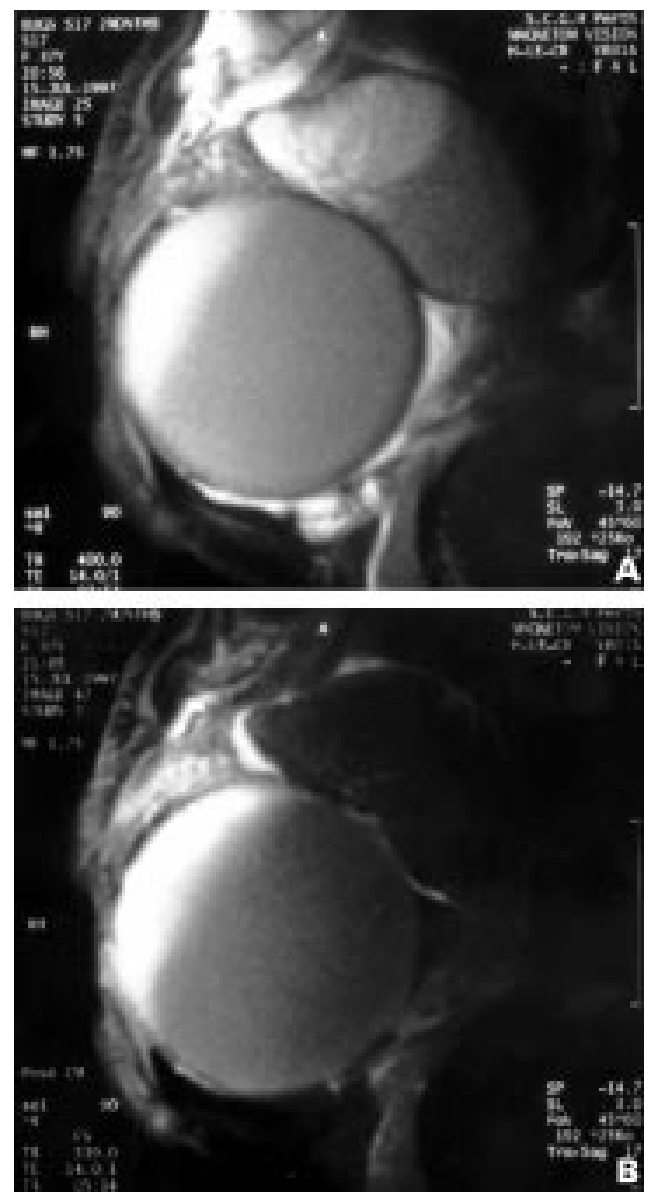

Figure 5 Magnetic resonance images of implant in situ at 2 months $(A)$ precontrast and $(B)$ after gadodiamide injection. Note enhancement due to vascularisation of the anterior hemisphere.

In the 3 week and 2 month explants, specks of calcium were seen in the sponges, which were well biocolonised with cells and blood vessels. There was a marked inflammatory reaction surrounding and within the sponges comprising polymorphs, macrophages, and plasma cells.

In one of the two prostheses explanted at 1 months, a large calcium deposit was seen in the sponge anterior hemisphere. However, the sponge region in both prostheses was well colonised with fibroblast-like cells and blood capillaries which were filled with red blood cells (Fig 4A) in the most heavily populated regions. The capillaries were lined with endothelial cells that were flat and normal looking, indicating that they were mature blood vessels. Some inflammatory cells were seen within the sponge and surrounding tissue. Mature collagen fibres were seen surrounding the cells in the pores of the sponge (Fig 4B).

In both 6 month explants, calcium deposits were seen in the sponge only as small deposits or tiny specks. The sponge regions were profusely populated by fibroblast-like cells in most areas, with prolific vascularisation. Some inflammatory cells were seen within the sponge and the surrounding tissue.

In the 4 month explant, tiny calcium deposits were noted in the sponge, which was well colonised with fibroblastic cells, blood capillaries, and inflammatory cells.
MAGNETIC RESONANCE IMAGING

The implant was very clearly defined and did not disrupt the image of other orbital structures. The anterior sponge hemisphere of the implant produced a high signal on both T1WI and T2WI. The posterior gel component produced an intermediate signal on T1WI and low signal on T2WI. Suture tracks were evident as linear areas of low signal within the sponge hemisphere.

Contrast enhancement in the sponge, particularly near the sites of muscle attachment but extending to the centre, was most marked in the early postoperative period and remained strong at 2 months (Fig 5). By 6 months, enhancement is less marked, and at 12 months is minimal or absent (Fig 6). Moderate enhancement was noted surrounding the posterior hemisphere of the implant at 2 months, suggesting that minimal encapsulation had occurred, but this was not seen after 2 months. There was no enhancement within the posterior hemisphere. 

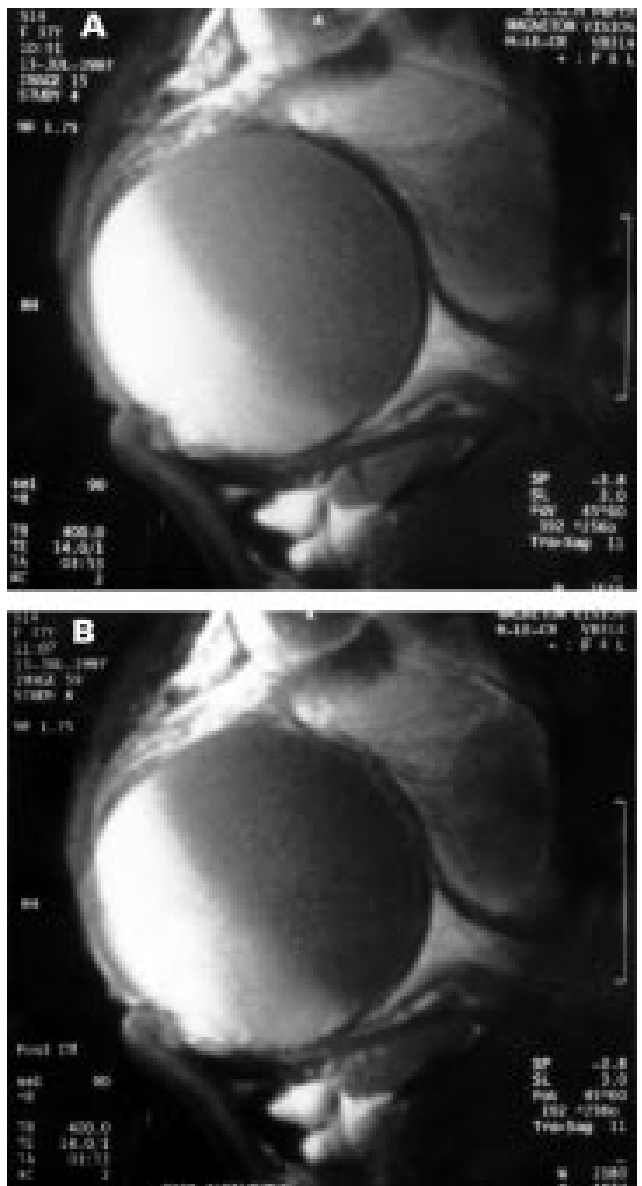

Figure 6 Magnetic resonance images of implant in situ at 12 months $(A)$ precontrast and $(B)$ after gadodiamide injection, showing minimal enhancement.

When scans were compared with corresponding photographs of hemisected specimens and micrographs, there was close agreement of features including muscle attachment, vascularisation, and presence or absence of a capsule. However, one specimen at 1 year showed a large deposit of calcium under the conjunctiva which had not been detected on MRI, and which appeared to be related to a lump of persistent suture material. The hemisected specimen, in its container of $2.5 \%$ glutaraldehyde, was subjected to CT scanning (Toshiba X-Press CT scanner; $1 \mathrm{~mm}$ thick contiguous slices, $120 \mathrm{kV}, 300 \mathrm{mAs}$ ) in the same plane as its original MR scan. This

Table 1 Summary of histological and imaging findings

\begin{tabular}{lll}
\hline Time implanted (no of specimens) & Histological findings & Imaging findings \\
\hline 3 weeks to 2 months (3) & Fibroblasts ++ & Enhancement ++++ \\
& Blood capillaries +++ & No calcium detected \\
& Inflammation +++ & Capsule + \\
& Calcium deposits + & \\
4 months (1) & Fibroblasts +++ & Enhancement +++ \\
& Blood capillaries ++ & No calcium detected \\
& Inflammation + & No capsule \\
& Calcium deposits + & \\
6 months (2) & Fibroblasts +++ & Enhancement ++ \\
& Blood capillaries ++ & No calcium detected \\
& Inflammation + & No capsule \\
& Calcium deposits + & \\
12 months (2) & Fibroblasts +++ & Enhancement + \\
& Blood capillaries + & Calcium deposit seen in one animal \\
& Inflammation + & No capsule \\
& Calcium deposits ++ & \\
& & \\
& & \\
& &
\end{tabular}
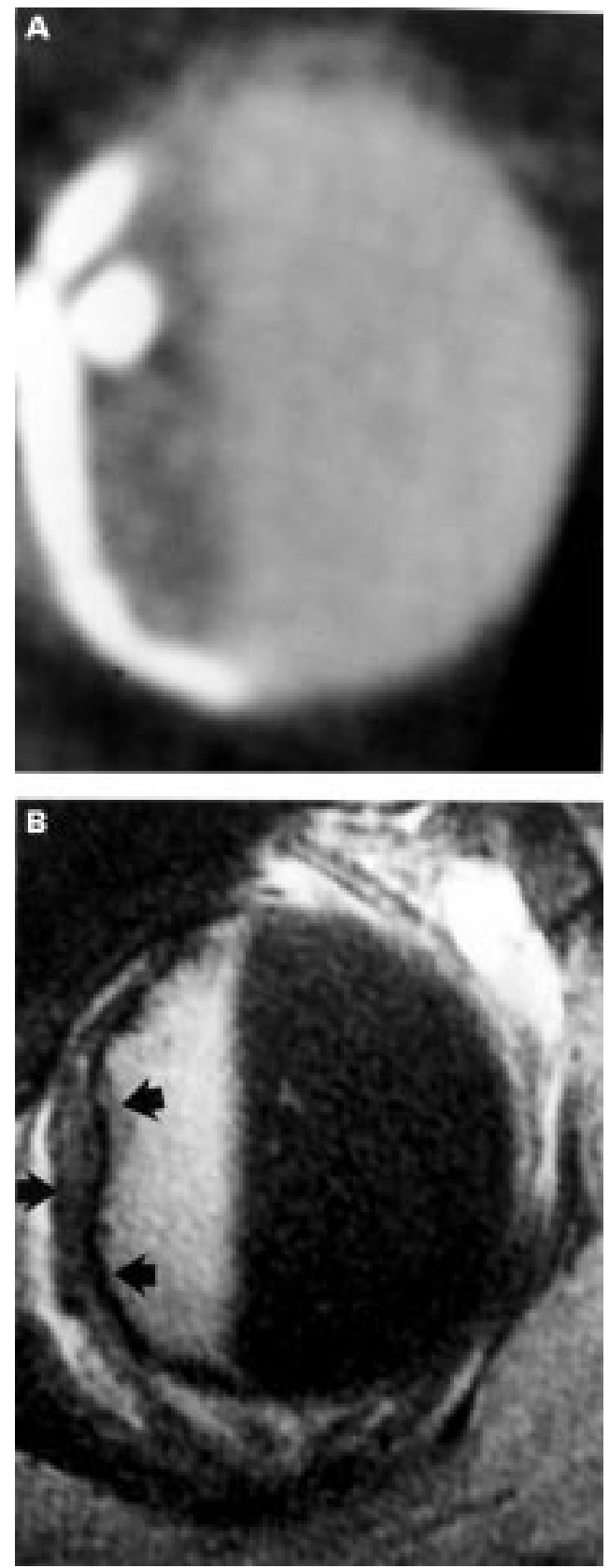

Figure 7 (A) In vitro CT scan showing an irregular white calcium deposit deep to the conjunctiva and (B) corresponding in vivo $M R$ scan, in which a corresponding area (arrows) is revealed as a hypodense region. The bright white artefact seen anteriorly to the implant is caused by a pooling of tear fluid.

revealed the deposit of calcium as a clearly defined white mass. On reviewing the corresponding MR scan, a hypodense area which corresponded in size and shape to the deposit seen on CT, was confirmed (Fig 7). MRI and histological findings are summarised in Table 1 .

\section{Discussion}

The expected advantages of this soft, biointegrable PHEMA implant in practice are better motility and reduced exposure when compared with solid implants, and motility comparable with that achieved by hydroxyapatite implants but with less risk of exposure, ${ }^{6-8}$ and without 
the requirement for coverage by scleral or alternative material before muscle attachment. PHEMA is inexpensive, and although other materials cheaper than hydroxyapatite, such as alumina $\left(\mathrm{Al}_{2} \mathrm{O}_{3}\right)$ or ceramic, have been proposed and assessed, they retain the disadvantage of physical hardness, requiring implant wrapping ${ }^{9}$ or placement of muscles within surface grooves.

It is important to understand the early and late tissue responses to an implanted biomaterial, and to know how appearances on imaging relate to healing or inflammatory changes. This is especially desirable for an orbital implant intended for long term retention and which may, in some cases, be implanted following enucleation for malignancy. Imaging artefacts must be distinguished from recurrences or infections. ${ }^{10}$ This study was therefore carried out both to gain familiarity with the appearance of the novel implant on MRI, and to study the process of biointegration by correlation of images with histological studies, to enable predictions of the implant's long term stability in vivo.

With the advent and increasing clinical use of porous biocolonisable orbital implants, chiefly of hydroxyapatite, increasing reliance has come to be placed upon imaging, ${ }^{11-19}$ especially where it is intended to drill into the implant to facilitate placement of a peg to allow coupling to the overlying cosmetic shell. CT has the disadvantages of ionising radiation, low contrast resolution, and artefacts related to scanning through the bony orbit. Technetium-99 bone scans indicate fibrovascular ingrowth but the low resolution, one dimensional image obtained tends to overestimate vascularisation within the central part of the implant. The area of vascularisation can be more accurately delineated with MRI, which was the method of choice for this study. The principles of orbital imaging using MRI have been well reviewed by Dortzbach et al..$^{21}$

Histological studies of alternative orbital implants have been performed in animal models, although generally not with images for comparison at the same end points. ${ }^{22}{ }^{23}$ In the early years of hydroxyapatite orbital implant implantation few detailed histological studies were available, obtained only after removal of an implant from a human patient. ${ }^{24}$ A giant cell inflammatory reaction to the hydroxyapatite has been reported to accompany fibrovascular invasion, which is most pronounced over window defects in the scleral shell used to cover the hydroxyapatite. ${ }^{25}$ In one study, quantitative CT appearances of a hydroxyapatite implant in rabbits demonstrated increased mineral density correlating with a fibrovascular and mixed cell inflammatory response up to 6 weeks. $^{26}$

We have previously demonstrated viable cellular colonisation of PHEMA sponges in vivo which increases with time, and that inflammatory cells represent a minority of the invading cells. ${ }^{27}$ In the present study a PHEMA orbital implant with a biocolonisable anterior hemisphere was placed for up to 1 year within the rabbit orbit and analysed by correlating contrast enhanced MRI with dissection and histology. The florid enhancement surrounding the sites of recti attachment seen on the early postoperative images reflects the initial hypervascular inflammatory response seen histologically. The reduction in the degree of enhancement over time indicates the transformation from a reactive, inflammatory tissue to quiescent, less vascular scar tissue. This is confirmed histologically at the later stages, where fibroblast dominant colonisation of the sponge hemisphere is seen. The maturity of the blood capillaries and collagen fibrils present several months after implantation suggest that stable long term retention of the implants can be expected.

Calcium deposits which were too small to detect on imaging were noted histologically as tiny specks within the sponge. They did not appear to increase in size over time nor to cause adverse effects. However, one animal at 12 months demonstrated a large calcium plaque associated with a suture remnant within the anterior sponge beneath the conjunctiva, which could be visualised on both MRI and CT scans. It is possible that such a large, hard deposit could eventually have caused conjunctival erosion in a manner similar to that occasionally reported with hydroxyapatite implants.

\section{Conclusion}

These MRI, light microscopy and TEM findings indicate a change over 1 year post implantation of a soft biocolonisable orbital implant from reactive inflammation to mature tissue integration. Secure attachment of muscles, which were sutured directly to the unwrapped implant, persists. Adhesions to the posterior, non-biocolonisable hemisphere, are minimal. These findings are consistent with the satisfactory clinical outcomes in this and a previous animal study, in which good cosmesis and movement were noted, and no implant extrusions occurred. A clinical trial is required to compare the performance of the PHEMA implant with alternative orbital implants in human patients.

The authors wish to thank Ms Donna Pead and staff of the Sir Charles Gairdner Hospital Animal House for their animal care and anaesthesia, and Mr Chris Barry for assistance with preparation of illustrations.

\footnotetext{
1 Hicks CR, Crawford GJ, Clayton AB, et al. The development of an orbital implant allowing direct muscle attachment. In: Suveges I, Follman P, eds. Societas Ophthalmologica Europaea, XIth Congress of the European Society of Ophthalmology. Bologna, Italy: Monduzzi Editore, 1997; Vol Ophthalmology

2 Hicks CR, Clayton AB, Vijayasekaran S, et al. Development of a PHEMA orbital implant allowing direct muscle attachment and tissue ingrowth. Ophthal Plast Reconstr Surg 1998; (in press)

3 Chirila TV, Vijayasekaran S, Horne R, et al. Interpenetrating polymer network (IPN) as a permanent joint between elements of a new type of artificial cornea. F Biomed Mater Res 1994;28:745-53

4 Jamell GA, Hollsten DA, Hawes MJ, et al. Magnetic resonance imaging versus bone scan for assessment of vascularization of the hydroxyapatite orbital implant. Ophthal Plast Reconstr Surg 1996;12:127-30.

5 Hicks CR, Clayton AB, Fitton JH, et al. Ocular socket prosthesis. Int Pat Appl No PCT/AU 97/00512, Aug 141997. 6 Nunnery WR, Heinz GW, Bonnin JM, et al. Exposure rate of Nunnery WR, Heinz GW, Bonnin JM, et al. Exposure rate of
hydroxyapatite spheres in the anophthalmic socket: histologic correlation and comparison with silicone sphere implants. Ophthal Plast Reconstr Surg 1993;9:96-104.
} 
7 Oestreicher JH. Treatment of exposed coral implant after failed scleral patch graft. Ophthal Plast Reconstr Surg 1994, failed scler $1110-13$.

8 McNab A. Hydroxyapatite orbital implants: experience with 100 cases. Aust NZ F Ophthalmol 1995;23:117-23.

9 Mawn LA, Jordan DR, Gilberg S, et al. Analysis of a new orbital implant made of alumina $\left(\mathrm{Al}_{2} \mathrm{O}_{3}\right)$ (Abstract). Inves Ophthalmol Vis Sci 1997;38(Suppl): 588 .

10 Summers CG. Calcification of scleral-wrapped orbital implant in patients with retinoblastoma. Paediatr Radio 1993;23:34-6.

11 Hamilton HE, Christianson MD, Williams JP, et al. Evaluation of vascularization of coralline hydroxyapatite ocular implants by magnetic resonance imaging. Clin Imaging 1992;16:243-6.

12 De Potter P, Shields CL, Shields JA, et al. The role of magnetic resonance imaging in the evaluation of the hydroxya-
patite orbital implant. Ophthalmology 1992;99:824-30.

13 Shields CL, Shields JA, De Potter P. Hydroxyapatite orbital implant after enucleation. Arch Ophthalmol 1992;110:333implat

14 Kazim M, Katowitz JA, Fallon M, et al. Evaluation of a collagen/hydroxyapatite implant for orbital reconstructive surgery. Ophthal Plast

15 Menzel C, Grunwald F, Busin M, et al. Vascularisation of ocular coralline hydroxyapatite implants. Eur $\mathcal{F}$ Nuclear Med 1994;21:1343-5.

16 Numerow LM, Kloiber R, Mitchell RJ, et al. Hydroxyapatite orbital implants. Scanning with technetium-99m MDP. Clin Nuclear Med 1994;19:9-12.

17 Toney MA, Heironimus JD 2d, Rivera D, et al. Scintigraphic evaluation of coralline hydroxyapatite ocular implants. Case reports on the use of planar and SPECT bone scintigraphy. Clin Nuclear Med 1993;18:50-2.
18 Dutton JJ. Coralline hydroxyapatite as an ocular implant. Ophthalmology 1991;98:370-7.

19 Goodall KL, Hill J, Talbot EM. Assessment of coral implant vascularisation utilising 3 dimensional single photon emission computerised tomography (3D SPECT) (Abstract). Invest Ophthalmol Vis Sci 1997;38(Suppl):587.

20 Dortzbach RK, Kronish JW, Gentry LR. Magnetic resonance imaging of the orbit. Part I. Physical principles. Ophthal Plast Reconstruct Surg 1989;5:151-9.

21 Dortzbach RK, Kronish JW, Gentry LR. Magnetic resonance imaging of the orbit. Part II. Clinical applications. Ophthal Plast Reconstr Surg 1989;5:160-70.

22 Rubin PA, Popham JK, Bilyk JR, et al. Comparison of fibrovascular ingrowth into hydroxyapatite and porous polyethylene orbital implants. Ophthal Plast Reconstr Surg 1994;10:96-103.

23 Goldberg RA, Dresner SC, Braslow RA, et al. Animal model of porous polyethylene orbital implants. Ophthal Plast Reconstr Surg 1994;10:104-9.

24 Shields CL, Shields JA, Eagle RC, et al. Histopathologic evidence of fibrovascular ingrowth four weeks after placement of the hydroxyapatite orbital implant. Am f Ophthalmol 1991;111:363-6.

25 Rosner M, Edward DP, Tso MOM. Foreign-body giant-cell reaction to the hydroxyapatite orbital implant. Arch Ophthalmol 1992;110:173-4.

26 Sires BS, Holds JB, Archer CR, et al. Histological and radiological analyses of hydroxyapatite orbital implants in rabbits. Ophthal Plast Reconstr Surg 1995;11:273-7.

27 Vijayasekaran S, Fitton JH, Hicks CR, et al. Cell viability and inflammatory response in hydrogel sponges implanted in the rabbit cornea. Biomaterials 1998; (in press). 